\title{
Tendências da vulnerabilidade às infecções pelo HIV e HCV entre internos da FEBEM avaliados em diferentes períodos - 1994 a 1995 e 2000 a 2001
}

\section{Vulnerability trend related to HIV and HCV infections among FEBEM's inmates evaluated in different periods 1994 to 1995 and 2000 to 2001}

\author{
Leila Strazza'1, Eduardo Massad², Heráclito B. Carvalho³
}

\begin{abstract}
Strazza L, Massad E, Carvalho HB. Tendências da vulnerabilidade às infecções pelo HIV e HCV entre internos da FEBEM avaliados em diferentes períodos - 1994 a 1995 e 2000 a 2001. Saúde, Ética \& Justiça. 2005;10(1/2):48-53.

RESUMO: Este estudo faz uma abordagem da condição do menor infrator, internos da FEBEM (Fundação Estadual para o Bem Estar do Menor de São Paulo) e sua situação de risco sob diferentes prismas. Apóia-se em dois estudos homogêneos desenvolvidos em momentos diferentes, onde avaliam a vulnerabilidade do menor infrator à infecção pelo HIV e HCV, nos períodos de 1994 a 1995 e 2000 a 2001.
\end{abstract}

DESCRITORES: Infecções por HIV/transmissão. Hepacivirus. Comportamento sexual. Drogas ilícitas/efeitos adversos. Drogas ilícitas/legislação \& jurisprudência. Saúde do adolescente institucionalizado. Transtornos relacionados ao uso de substâncias. Grupos de risco.

INTRODUÇÃO

A síndrome da imunodeficiência adquirida (aids) não é um problema puramente médico, pois traz consigo muitas questões ligadas à cultura que devem, não apenas serem respeitadas, como também, avaliadas e trabalhadas na sua essência que envolvem a cidadania, a sexualidade, o medo, os preconceitos, o gênero, a política social e econômica de cada país, cidade, estado ou região ${ }^{20}$.

Se, para os locais mais desenvolvidos e favorecidos, é complexo abordar tais problemas, como fica então, essa abordagem em um país em desenvolvimento? As políticas de saúde, social e econômica têm sido capazes de dar proteção aos

1 Pesquisadora Doutora - LIM 01- Faculdade de Medicina, Universidade de São Paulo - FMUSP.

2 Professor Titular da Disciplina de Informática Médica e Chefe do Departamento de Medicina Legal, Ética Médica e Medicina Social e do Trabalho da Faculdade de Medicina, Universidade de São Paulo - FMUSP. email: edmassad@usp.br.

3 Professor Doutor, Departamento de Medicina Preventiva da Faculdade de Medicina, Universidade de São Paulo LIM 39 - FMUSP. e-mail: heracc@ usp.br

Endereço para correspondência: Leila Strazza. Faculdade de Medicina Legal da Universidade de São Paulo. Instituto Oscar Freire. R. Teodoro Sampaio, 115 - Disciplina de Informática Médica - LIM 01 - 2ํAndar. São Paulo - SP. CEP 05405-00. E-mail: strazza@usp.br 
cidadãos, em especial ao menor abandonado²? Este, quase que, de uma forma determinística, termina como um temido infrator. Quando enfocamos o menor, mais precisamente, o adolescente, sabemos que este apresenta uma capacidade capaz de assimilar os impactos projetivos de pais, irmãos, sociedade, ou seja, é como se fosse um receptáculo que se encarrega dos conflitos alheios, principalmente, os doentios do meio em que vive ${ }^{20}$. Muitos fatores podem contribuir para que adolescentes passem a ter um comportamento antisocial, tornando-os delinqüentes. A própria adolescência é tida como época complexa da vida de uma pessoa, quando situações positivas e negativas convivem intensamente. Essa afirmação remete a uma comparação com a sociedade, que projeta suas falhas nos chamados "excessos de juventude" e que os responsabiliza pela delinqüência, drogas, prostituição ${ }^{20}$.

Freud ${ }^{13}$, em 1958, afirmou: "É muito difícil assinalar o limite entre o normal e o patológico na adolescência". Winnicott ${ }^{24}$ enriquece a psicanálise com sua forma de discutir a delinqüência como uma defesa anti-social organizada que é sobrecarregada com ganho secundário e reações sociais. Esclarecendo sobre a tendência anti-social de Winnicott ${ }^{24}$, que diz que existe um "complexo de privação" que ocorre quando uma criança sofre de privação, ou seja, quando lhe faltam certas características que lhe são essenciais da vida familiar, que a faz expor um comportamento antisocial. Em virtude desta tendência, a pessoa será considerada como desajustada ou levada ao tribunal como incontrolável. Porém, na delinqüência a criança pode ser mandada para um reformatório. A repetição destes atos é denominada reincidência.

A tendência anti-social implica na esperança, na compreensão do ato. É, fundamental, no tratamento, o momento ato anti-social, ele não deve ser desperdiçado.

Segundo Winnicott ${ }^{24}$, quando existe esta tendência anti-social há um desapossamento que não quer dizer carência, pois no caso é entendido como uma perda de algo bom, positivo na experiência da pessoa até aquela data em que foi retirado. Essa retirada foi por um período maior do que se pode manter viva a lembrança da experiência boa. A privação inclui o ponto exato do trauma sofrido e a persistência da condição traumática.

Voltando à delinqüência como um ato antisocial $^{25}$ que pode levar ao crime, diremos que a atitude sentimentalista em relação ao crime leva ao ódio recalcado e esse recalcamento não é saudável. Lembremos que o crime produz sentimentos de vingança pública, que redundaria em algo perigoso, sendo que o mesmo, é afastado num tribunal por um magistrado que, não apenas, estabelece a sentença, como também, a chance de um tratamento humano ao infrator, que é uma pessoa doente. Dito isto Winnicott ${ }^{25}$, em 1995, torna-se, mais claro, ao afirmar que o criminoso retém uma doença psicológica que deve ser tratada, pois é apresentada como recuperável.

Numa família onde marido e mulher assumem a responsabilidade de uma criança, somos cientes de que os estágios iniciais do desenvolvimento emocional são conflitantes e repletos de desintegrações potenciais, o qual torna a criança um ser destrutivo, mas o lar a ajudará a suportar toda esta desorganização e faz com que ela vá brincar, saudavelmente ${ }^{26}$.

Na delinqüência desenvolvida, a situação fica difícil, neste momento, pois a criança busca um protetor para recuperar seus impulsos primitivos de amor, o seu desejo de recuperar-se, mas, ela está só, o que, por conseguinte, fará com que cada vez fique mais deprimida e despersonalizada, incapaz de sentir a realidade das coisas, exceto a realidade da vida. Só lhe resta o ato anti-social que, nada mais é, do que um pedido de socorro e controle às pessoas fortes, amorosas e confiantes ${ }^{25}$.

Pesquisas feitas por Dryfoos ${ }^{9}$, em 1990, já identificavam que um em cada quatro adolescentes corria o sério risco de tornar-se um adulto irresponsável, pois os mesmos não estavam adquirindo habilidades, suficientes e necessárias, à participação do sistema educacional e o cumprimento à força de trabalho. A vida familiar desses jovens era limitada, tornando-os incapazes de serem pais responsáveis. Dryfoos ${ }^{9}$, em 1990, afirmou que uma nova classe estava surgindo: "os intocáveis" pessoas analfabetas desligadas da escola, deprimidos, propensos ao uso excessivo de drogas, pais de filhos indesejados, e, infelizmente, que poderiam ligar-se à vida criminal. Quem é a sociedade, neste momento, para este grupo?

Uma condição externa vivida por esses adolescentes, com influência no processo ideológico e de um ideal, que antes era baseado na virtude, hoje, o que mais conta, é o sucesso, não importando de onde ele seja proveniente e quais os meios necessários exigidos para alcançá-lo. A sociedade em que vivemos, faz com que o adolescente que está tentando planejar sua vida, controlar suas mudanças, adaptar-se ao mundo e suas necessidades imperiosas entre no seu conflito pessoal, se depare com as frustrações diárias do cotidiano. Esta, talvez, seja a forma de explicar os seus desejos e necessidades de reformas sociais. 
Reprimir um jovem com severidade e violência acaba criando um distanciamento maior e um agravamento de conflito, que colabora para sua agressão social. As grandes cidades, os centros mais dinâmicos do capitalismo no Brasil, são espaços onde a riqueza e a opulência confronta-se com a maior miséria, ficando claro o porquê das maiores formas de violência ${ }^{20}$.

De acordo com a Lei n. 7.244 , de 7 de novembro de 1984 sancionada pelo Presidente da República: “...o ser humano não pode ser considerado de forma isolada, mas sempre dentro de um contexto mais dilargado, isto é, no cerne de um complexo numeroso visualizado pela sociedade, pois é parte desse todo" (p. 31) ${ }^{14}$.

Esses adolescentes carregam uma carga comum, bastante parecida: genitores desconhecidos, criados pela caridade do outro e impelidos, muito cedo, para a sarjeta, alguns permaneceram sob o mesmo teto de ódio permanente entre seus pais; assistiram a cenas protagonizadas pelo uso abusivo do álcool, imitaram pais criminosos, sofreram castigos físicos ${ }^{1,20}$, expuseram seus corpos a maior miséria social e abandono econômico que termina por conduzi-los à criminalidade e à maior exposição a doenças sexualmente transmitidas como a aids ${ }^{19}$.

Quanto a esta herança, acreditamos na predisposição geral da transmissão desenvolvida, com a influência do meio vivido pelo indivíduo, podendo seguir um paralelo, da pessoa subnutrida estar mais predisposta a contrair tuberculose pulmonar, não somente por ela, mas por tudo aquilo que a cerca ${ }^{20}$

Diante desse quadro, onde esse adolescente especial vive exposto a um mundo tão desigual, desenhamos este estudo para avaliar a vulnerabilidade destes adolescentes à infecção do HIV e HCV ao longo de um período de cinco anos. Independente da identificação do risco medido pelo comportamento inadequado, muitas questões cercam esse quadro, onde as estratégias de controle são desejáveis.

No Brasil, na época em que os dois estudos foram desenvolvidos, o número de menores infratores era estimado em 2,7 por 100 mil habitantes ${ }^{20}$.

\section{MÉTODOS}

Tipos de estudo: foram realizados dois estudos em São Paulo: Strazza ${ }^{20}$, em 1995 e Carvalho et al. ${ }^{6}$, em 2001. Estudos do tipo transversais de prevalência para a infecção HIV e HCV com abordagem metodológica bastante semelhante.

Os estudos utilizaram questionários de comportamento de risco tanto sexual como de uso de drogas. Os resultados foram agrupados em categorias que pudessem ser tratadas de forma homogênea. Embora ambos tenham sido feitos na Fundação para o Bem-Estar do Menor de São PauloSP (FEBEM) na cidade de São Paulo, os locais onde dois estudos foram realizados foram diferentes $\mathrm{e}$ estão descritos abaixo, quando da época do estudo:

$>$ Dezembro de 1994 a março de 1995 - o $1^{0}$ estudo aceitou o convite da Secretaria do Bem Estar Social daquela época e realizou este trabalho no Quadrilátero Tatuapé - 12 unidades - (masculino) e Rodovia dos Imigrantes três casas (feminino) unidades com capacidade para 110 internos;

$>$ outubro de 2000 a janeiro de 2001 - o $2^{\circ}$ estudo realizou este trabalho em duas unidades Unidade Parada de Taipas - (feminino) e Unidade Raposo Tavares - (masculino) - unidades com capacidade para 50 internos.

Instrumento e coleta de dados No $1^{\circ}$ estudo os adolescentes responderam a um questionário realizado através de um questionário piloto feito anteriormente e no $2^{\circ}$ estudo eles responderam a um questionário semi-estruturado, cujo núcleo básico foi constituído pelo questionário padrão de estudo multicêntrico de1994 da OMS ${ }^{18}$ utilizado em estudos desenvolvidos no Brasil ${ }^{3,4,5}$. Em ambos os jovens foram abordados pela técnica "face-to-face"

Análise estatística: Foi utilizada análise paramétrica do tipo teste $t$ de Student para as variáveis com distribuição normal e teste de qui quadrado e $\mathrm{H}$ de Kruskal-Wallis para as variáveis não paramétricas. Foram também utilizados os pacotes estatísticos EPIINFO versão $6.0^{8}$ e SPSS versão $10.0^{16}$ para elaboração dos bancos de dados e execução das análises descritivas e analíticas.

Análise laboratorial: Utilizou-se, para triagem diagnóstica de HIV, testes imunoezimáticos para pesquisa de anticorpos (Elisa) anti-HIV tipos 1 e 2 de terceira geração, da marca Ortho Diagnostics. Os casos positivos tiveram resultados confirmados pela técnica Western Blot, anti-HIV tipo 1, da marca Sanofi-Pasteur. O diagnóstico da infecção pelo vírus da hepatite $C$ foi determinado por testes Elisa antiHCV (vírus da hepatite C) de terceira geração, da marca Ortho Diagnostics.

Os resultados de todos os exames foram entregues individualmente pela equipe médica da própria FEBEM, por decisão de seus diretores.

Aspectos éticos: Foi dada especial atenção a todos os aspectos éticos envolvidos nesses estudos. Ambos os estudos foram submetidos e aprovados pela Comissão de Ética Médica do HCFMUSP, nos momentos devidos.

Saúde, Ética \& Justiça, São Paulo. 2005;10(1/2):48-53. 


\section{RESULTADOS}

Foram obtidos os diagnósticos sorológicos para a infecção pelo HIV e HCV nos diferentes grupos, no período compreendido entre 1994 e 2001.

No estudo de Strazza $^{20}$ foram entrevistados 87 adolescentes do sexo feminino e 1122 adolescentes do sexo masculino e no estudo de Carvalho et al. ${ }^{6}$ participaram 45 meninas e 38 meninos.

Ambos os estudos entrevistaram os adolescentes que estavam presentes na unidade naquele dia. na Tabela 1

O perfil da amostra estudada foi sumarizado

Tabela 1. Características sócio-demográficas de internos da FEBEM, em dois estudos conduzidos em São Paulo entre 1994 a 1995, (estudo 1) e 2000 e 2001, (estudo 2).

\begin{tabular}{l|l|l}
\hline Variáveis & Estudo 1 & Estudo 2 \\
\hline Sexo & & \\
\hline Masculino & 1122 & 38 \\
Feminino & 87 & 45 \\
\hline Idade em anos & & \\
\hline Masculino & 16,21 & 17,5 \\
Feminino & 15,95 & 16,3 \\
Estudo formal & $\begin{array}{l}\text { primeiro grau } \\
\text { incompleto }\end{array}$ & $\begin{array}{l}\text { primeiro } \\
\text { grau incompleto }\end{array}$ \\
\hline
\end{tabular}

Fonte: \# Strazza et al. ${ }^{20, \# ~ \#, ~ C a r v a l h o ~ e t ~ a l . ~}{ }^{6 ;}$ * Fundação do Bem Estar do Menor
O padrão de comportamento sexual foi semelhante em ambos os estudos nos seguintes tópicos:

$>$ A grande maioria destes indivíduos 95\% referiu já ter tido relação sexual com uma média de mais de 3 parceiros nos últimos 12 meses;

$>$ a prostituição, isto é, troca de dinheiro, bens ou droga por sexo, esteve presente nos discursos dos meninos e meninas;

$>$ foi relatado já ter tido alguma doença sexualmente transmissível em $13 \%$ dos internos.

A prevalência de infecções pelo HIV e HCV (Tabela 2) ainda é presente em ambos estudos e maior que o da população geral ${ }^{17}$.

Carvalho et al. ${ }^{6}$ observaram associação do HCV com uso de drogas injetáveis $(x 2=15,3, p=$ $0,00009)$ e Strazza et al. ${ }^{20}$ observaram que a via parenteral está fortemente indicada como fator de vulnerabilidade para a transmissão do HIV entre os adolescentes do sexo masculino, com odds ratio de 20,7 para o uso de drogas injetáveis.

Quanto ao uso de droga injetável foi observado em Strazza ${ }^{20}$ e Strazza et al. ${ }^{21,22,23}$ que $15 \%$ das meninas e $6,5 \%$ dos meninos tinham referido uso da droga por essa via, enquanto que em Carvalho et al. ${ }^{6}$, apesar de ter apresentado uma redução da freqüência, apresentava a idade media de primeiro uso que foi de 14 anos.

Tabela 2. Prevalência de HIV e HCV de internos da FEBEM, em dois estudos conduzidos em São Paulo entre 1994 a 1995, (estudo 1) e 2000 e 2001, (estudo 2).

\begin{tabular}{l|l|l|l|l|l}
\hline Estudo & Ano da Pesquisa & Sexo & N & \% HIV & \% HCV \\
\hline Estudo 1 & 1994 & Feminino & 87 & 10,3 & 3,4 \\
& 1995 & Masculino & 1122 & 2,6 & 5,9 \\
Estudo 2 & 2000 e 2001 & Feminino & 45 & $1,0 \%$ & $6,0 \%$ \\
& 2000 e 2001 & Masculino & 38 & $0 \%$ & $6,0 \%$ \\
\hline
\end{tabular}

Fonte: \# Strazza et al ${ }^{20, \# \# ~ C a r v a l h o ~ e t ~ a l ~}{ }^{6}$; * Fundação do Bem Estar do Menor

Tabela 3. Prevalência de uso de droga ilícita dentro e fora da FEBEM*, entre 1994 a 1995.

\begin{tabular}{l|c|c|c|c}
\hline \multirow{2}{*}{ Droga } & \multicolumn{2}{|c|}{ Dentro } & \multicolumn{2}{c}{ Fora } \\
\cline { 2 - 5 } & Feminino & Masculino & Feminino & Masculino \\
\hline Maconha & $8,3 \%$ & $11,5 \%$ & $87,4 \%$ & $83 \%$ \\
Cocaína & $1,2 \%$ & $2,0 \%$ & $64,4 \%$ & $63 \%$ \\
Crack & $6,0 \%$ & $1,6 \%$ & $69,0 \%$ & $55 \%$ \\
Haxixe & $1,2 \%$ & $1,0 \%$ & $41,4 \%$ & $37 \%$ \\
LSD & $0 \%$ & $0,3 \%$ & $9,2 \%$ & $3,5 \%$ \\
\hline
\end{tabular}

Fonte: Strazza et al. ${ }^{20}$; * Fundação do Bem Estar do Menor
Tabela 4. Prevalência de uso de droga ilícita fora da FEBEM $^{*}$, entre 2000 e 2001.

\begin{tabular}{llll}
\hline Droga & Idade média em anos & ICI $^{\#}$ & ICS $^{\#}$ \\
\hline Inalantes & 11,3 & 8 & 15 \\
Álcool & 11,8 & 12 & 15 \\
Tabaco & 11,2 & 11 & 15 \\
Cocaína & 13,5 & 10 & 17 \\
Maconha & 13,4 & 12 & 16 \\
Crack & 14,0 & 10 & 15 \\
\hline Fonte: Carvalho et al. ${ }^{6 ;}{ }^{*}$ Fundação do Bem Estar do Menor
\end{tabular}

Fonte: Carvalho et al. ${ }^{6}$; ${ }^{*}$ Fundação
Intervalo inferior; \# \# Intervalo superior 
As semelhanças dos dois estudos existem quanto ao uso de drogas ilícitas (Tabelas 3 e 4) dentro e fora da FEBEM, chamou atenção, no entanto, que no estudo de Carvalho et al. ${ }^{6}$ referente ao "crack" com conseqüências à problemas sociais e jurídicos, foi observado que todos (100\%) dos que usaram o "crack", tiveram algum envolvimento com violência, sendo que $72 \%$ desta violência estava associado à droga. A violência era identificada por: roubo, brigas, briga verbal, tráfico, roubo para obter droga, receber ameaça, receber ameaça de morte, vítima de abuso sexual, autor de abuso sexual.

\section{DISCUSSÃO}

Este grupo, em especial, usa drogas muito intensamente que é mais um agravante de sua vulnerabilidade tanto a estes problemas citados como às infecções estudadas.

Em países em desenvolvimento, onde estes jovens proliferam-se cada vez mais nas ruas das áreas metropolitanas, a prevenção acaba sendo uma prioridade para a saúde pública.

Evidências apontam para um aumento do consumo de "crack" no Brasil, a partir de $1990^{10,15}$, e a uma adesão dos usuários de drogas injetáveis à nova droga ${ }^{6}$, é pertinente supor a possibilidade de existir um impacto na prevalência de infecções de transmissão por via parenteral, caso haja uma mudança, no hábito destes usuários, no sentido da troca, isto é, uso do "crack" pela droga injetável ${ }^{4,6}$.

Eles vivem muito próximos de várias formas de violência que precisam ser melhor entendidas, porém existem situações em que este menor infrator é vulnerável à transmissão do HIV e nem ao menos Ihe é permitido decidir pelo sexo seguro como demonstra abaixo o recorte do discurso de um adolescente da FEBEM $^{20}$ com algumas considerações a respeito:

- "a gente dorme juntos porque tem medo que no meio da noite a polícia chegue, bata na gente e jogue dentro do camburão" (p. 27);
$>$ pode-se dizer que esses adolescentes escolheram dormir juntos e, assumir um comportamento de risco?;

$>$ esse comportamento é inadequado?;

$>$ onde está o risco a ser combatido: na infecção do HIV ou na violência exposta?;

$>$ qual a alternativa para aquele momento?

Não se aceita a situação de risco como condição constitutiva da pobreza, miséria e do desamparo da política social, mas sim, estar frente à uma situação real de vida onde o risco se faz presente e que mostra a vulnerabilidade destes jovens, não somente ao HIV, mas, sim, à violência da realidade da vida que eles estão expostos. Freire ${ }^{11,12}$ lembra que a leitura crítica da realidade se constitui como um instrumento necessário de resgate à cidadania do indivíduo que luta nos movimentos sociais, não apenas, pela melhoria da qualidade de vida, mas também, pela transformação social.

Como evitar então, o comportamento de risco? O sexo é um dos prazeres que esta população têm e sem dúvida, é o escape que eles tem da realidade que Ihes é muito cruel, ou melhor, suas vidas. Eles têm muitos problemas em relação à pobreza que fazem sombra à aids. O que realmente preocupa-os é saber onde irão dormir à noite e se acordarão com vida ${ }^{19,21,22,23}$.

\section{CONCLUSÃO}

Além de ficar evidenciada a associação do "crack" com briga e assalto para obtenção de drogas, foi observado a associação do HCV com uso de drogas injetáveis $(x 2=15,3, p=0,00009)$ e que a via parenteral está fortemente indicada como fator de vulnerabilidade para a transmissão do HIV entre os adolescentes do sexo masculino, com odds ratio de 20,77 para o uso de drogas injetáveis.

Porém, fica muito difícil pensar na vulnerabilidade somente observando o comportamento sem levar em conta o papel da inserção do adolescente na sociedade.

Strazza L, Massad E, Carvalho HB. Vulnerability trend related to HIV and HCV infections among FEBEM's inmates evaluated in different periods 1994 to 1995 and 2000 to 2001. Saúde, Ética \& Justiça. 2005;10(1/2):48-53.

ABSTRACTS: This study is focused on the frame of disadvantaged youth, under internment in FEBEM (Fundação Estadual para o Bem Estar do Menor de São Paulo) concerning the risk under different prism. It is supported by two homogenous studies that were developed in different periods. They evaluated the vulnerability of these subjects to HIV and HCV infections in the periods from 1994 to 1995 and from 2000 to 2001.

KEY WORDS: HIV Infections/transmission. Hepacivirus. Sexual behavior. Street drugs/adverse effects. Street drugs/ legislation \& jurisprudence. Health of institutionalized adolescents. Substance-related disorders. Risk groups.

Responsável pelo projeto (1994/95): Prof.Dr. Eduardo Massad; Pesquisadora principal: Dra. Leila Strazza; Responsável pelo projeto (2000/01): Prof.Dr. Heráclito B.Carvalho;

Agradecimentos: Esses trabalhos foram financiados pela FAPESP, Fougarty Foundation, Pronex, LIM 01. 


\section{REFERÊNCIAS}

1. Butler T, Robertson P, Kaldor J. Syphilis in New South Wales (Australia) prisons. Int J STD AIDS. 2001;12(6):376-9.

2. Carvalho HB. A epidemia de aids em usuários de drogas injetáveis em algumas grandes cidades brasileiras. In: Vianna ALD, Elias PEM, Ibañes N, org. Proteção social - dilemas e desafios. São Paulo: Hucitec, 2005. v. 1, p. 315-34

3. Carvalho HB. Dinâmica de transmissão do HIV entre usuários de drogas injetáveis na cidade de Santos, São Paulo, Brasil [tese]. São Paulo: Faculdade de Medicina da Universidade de São Paulo, Departamento de Patologia; 1995.

4. Carvalho HB. "Relatório do Projeto Brasil - 1996: soroprevalência e seus determinantes para infecção pelo HIV em usuários de drogas injetáveis em 7 cidades brasileiras. Relatório Técnico, PNDST/AIDS. Brasília: Ministério da Saúde; 1997. [mimeo]

5. Carvalho HB, Mesquita F, Burattini MN, Massad E. HIV and infections of similar transmission patterns in a drug injectors community of Santos, Brazil. J Acquir Immune Defic Syndr Human Retrovirol. 1996;(2):8492.

6. Carvalho HB, Seibel SD, Burattini MN, Massad E, Reingold A.Vulnerabilidade às infecções pelo HIV, hepatites $\mathrm{B}$ e $\mathrm{C}$ e sífilis entre adolescentes infratores institucionalizados na cidade de São Paulo, Brasil. J Bras Doenças Sex Transm. 2003;15(3):41-5.

7. Catania JA, Kegeles SM, Coates TK. Towards an understanding of risk behavior: an AIDS risk reduction model (ARRM). Health Educ Q. 1990;17(1):53-72.

8. Dean AD, Dean JA, Burton, JH, Dicker RC. Epiinfo, revised version 6.04: a word processing, data base, and statistics program for Public Health on IBM compatible Miicrocomputers. Atlanta, Georgia: C D C; 1996.

9. Dryfoo S. 1990. In: Straus MB. Violência na vida dos adolescentes. São Paulo: Ed. Best Seller; 1994.

10. Mortalidade por "crack" é maior em São Paulo. São Paulo: Folha da Manhã, 24 jul. 1996. Folha de São Paulo.

11. Freire P. A educação na cidade. São Paulo: Cortez; 1991.

12. Freire P. Pedagogia do oprimido. 7a ed. Rio de Janeiro: Paz e Terra; 1979.

13. Freud S. Adolescence - the psychoanalytic study of the child. New York: International University Press; 1958 . v.13.

14. Frigini R. Juiz de Direito da $1^{\text {a }}$ Vara da Comarca de
São João da Boa Vista - Pequenas causas. Leme: Livraria e Editora de Direito; 1995.

15. Gregori MF. O espaço da viração. Estudos contemporâneos. Rio de Janeiro: Senac; 1995. v.1, p.59-63.

16. Marija J, Norusis J. SPSS advanced statistics 6.1 Statistical Software, SPSS Inco., Chicago, IL; 1994.

17. Brasil. Ministério da Saúde. Epidemia de AIDS no Brasil: atualização [citado em June 2000]. Available from: http://www.aids.gov.br/udtv/tabelan/epidemia.htm

18. World Health Organization. Multy-city study on drug injecting and risk of HIV infection - Programme on Substance abuse. WHO/PSA/94.4. Final report of the WHO/PSA. Genebra; 1994.

19. Strazza L, Massad E, Burattini MN, Zanetta D, Carvalho HB, Azevedo RS. A vunerability approach to HIV / Aids infection in disadvantage female adolecents of São Paulo, Brasil. In: Abstract book of $8^{\text {th }}$ International Conference On The Reduction Of Drug Related Harm, Paris, França; 1997. p. 151.

20. Strazza L. Diferenças de gênero e risco para a infecção pelo HIV em adolescentes internos na Fundação Estadual para o Bem Estar do Menor de São Paulo-FEBEM [dissertação]. São Paulo: Faculdade de Medicina da Universidade de São Paulo; 1999.

21. Strazza L, Massad E, Burattini MN, Carvalho HB, Azevedo RS. The prevalence of HIV and risk-taking behaviors among disadvantaged male and female in São Paulo, Brazil. In: Abstract book of XI International Conference on AIDS, Vancouver; 1996. v.1, p.256.

22. Strazza L, Massad E, Burattini MN, Carvalho HB, Azevedo RS. HIV infection among disadvantaged youth of São Paulo, Brazil. In: Abstract book of 12th World AIDS Conference, Itália; 1998. p.119.

23. Strazza L, Massad E, Burattini MN, Carvalho HB, Azevedo RS. HIV infection and related risk behaviours in a disadvantaged youth institution of São Paulo Brazil. In: Program supplement of XIII International AIDS Conference, Durban - África do Sul; 2000. v.1, p.26.

24. Winnicott DW. Da pediatria à psicanálise. 2a ed. Rio de Janeiro: Livraria Francisco Alves; 1982.

25. Winnicott DW. Alguns aspectos psicológicos da delinqüência juvenil, privação e delinqüência. São Paulo: Martins Fontes; 1995.

26. Winnicott DW. O brincar e a realidade. Rio de Janeiro: Imago; 1975. 